\title{
NOTE SUR LES CIMETIÈRES EN TUNISIE
}

\author{
rar \\ ABDEL-HAKIM EL GAFSI
}

C'est au cours de la prospection effectuée, dans le cadre de la preparation de la carte nationale des sites archéologiques et des monuments historiques, que nous avons pu evaluer l'ampleur, l'importance, la diversité et la richesse des champs funéraires. Mais nous allons nous limiter dans le cadre de ce travail aux cimetières musulmans (1). Par ailleurs, nous n'allons pas evoquer un certain nombre de problèmes importants à savoir, la mort, les pratiques funeraires, les enterrements... (2).

\section{I lieux de sepulture}

Il convient de rappeler que les mosquées, zaouias (mausolees) sont des lieux de sepulture favoris. Mais, un sondage rapide dans les chroniques tunisiennes, nous a permis de constater qu'il se trouve d'autres lieux: les palais (3), les ribats (couvents et forteresses) (4), les maisons (5) et les medersas (collège secondaire et universite (6).

\section{A les mosquées}

Il importe de mentionner qu'une foule de savants ont preféré le masğid

(1) Les cimetiéres chretiens et juifs n'entrent pas dans le cadre de cetravail. Pour se renseigner, sur le plan juridique consulter, le journal officiel tunisien. $N{ }^{\circ} 58$ de 1884.

(2) Entre autres, nous savons que les princes pouvaient suivre le cortège funèbre, voir à ce propos Ar rassà Fahrast, Tunis, librairie al Atika, 1967, p. 184.

(3) Al Massúdī, al ظulāșa, Tunis, Picard, 1323, p. 45.

(4) id., p. 48. Zbiss, S.-M., Inscriptions de Monastir, Tunis, INAA, 1960, p. 1.

(5) Id., Note sur les cimetières musulmans de Tunis, extrait du $70^{e}$ congrés de l'A.F.A.S., Tunis, mai 1951, p. 2. Al kinåni, Takmil, editè par Annabi, M, Tunis, al Atika, 1970, p. 98, 103.

(6) Ben Mami, B., Naḍra ḥawla ar turub, RHM, 33-34, 1984, p. 29. C'est le cas de la medersa d'lbn Tafarkin et du Kåid Nabīi. 
aș șafsāfa dans le quartier Nafāt, comme lieu d'inhumation (7) ou dans la mosquée el ksar (Bab Menara) (8) ou dans les mosquées Sidi Mardoum (Rue Sidi Mardoum), Sidi Ben Ziad (La Kasbah (9) ou al Kubba (Rue Tourbet el Bey (10).

\section{B les zaouias (mausolées)}

Oratoire, siège de confrerie religieuse, lieu d'anseignement, les zaouias propagent les doctrines mystiques du soufisme; elles pullulent dans les villes. Par conséquent c'est un foyer de spiritualité, de bénédiction (baraka). C'est pourquoni la population prefère étre ensevelie non seulement au milieu des siens mais aussi auprés d'un personnage réputé pour sa sainteté et sa dévotion (wali ou saint). En général chaque saint est enterré dans sa zaouia (11). C'est ainsi que dans le quartier de Bab al Jariza, à Tunis, se trouve encore la mausolée de Sidi Ali al andalousi (12). La famille Ben Achour d'origine andalouse (13) prefère la zaouia de sidi Ali zouawi du coté de Bab Menara (14) alors que la zaouia de sidi Battach (rue sidi ali azzouz) abrite entre autres mahammad al Hjaiej al andaiousi (15).

Signalons, par ailleurs que la zaouia de sidi Kasim az zelliji qui a servi de refuge aux moriscos andalous abrite la tombe du dernier souverain hafside, Hmida, contemporain de Charles Quint (16).

Fort evidemment ces mausolées se carecterisent, sur le plan architectural, par una architecture particulière. En effet, généralement chaque zaouia comporte una chambre funeraire abritant la tombe du saint cachée sous un catafalque (Tābut) en bois ouvragé autour duquel se placent les ex-votos: étendards, etoffes, tapis, textes sacrés, images de la Mecque; cette tombe est séparée du reste de la chambre par une grille de bois en moucharabie.le massif cubique de la chambre est couvert d'une coupole hémispherique construite en pots de terre cuite (chukkāf). La zaouia comprend aussi une mosquée et un kouttab (école coranique).

\section{C les tourbas (caveaux de famille)}

Les tourbas etaient reservées à une élite privilégiée; elle se trouvent soit

(7) Daoulatli, A., Tunis sous les Hafsides, Tunis, INAA, 1976, p. 169.

(8) Hugaa, H, Dayl, edité par Maamouri, T., Tunis, Maison arabe du livre, 1975, p. 180. Ben el khodja, M., Tarīh, edité par Ben Hadj yahia, et Sahli, H., Beyrouth, Dar al Gharb el islami, 1985, p. 173.

(9) Ben el khodja, M., op. cit., p. 248, 249.

(10) Ar rassac, op. cit., p. 15

(11) Al Kinani, op. cit., p. 40, 56, 101, 102,..

(12) Epalza, M., Petit, R., Etudes sur les moriscos andalous en Tunisie, Madrid, Intituto Hispanoárabe de Cultura, 1973, p. 268.

(13) Zbiss, S.-M., Gafsi, A., Boughanmi, M., Epalza, M., Etudes sur les morisques andalous, Tunis, INAA, 1983, p. 15.

(14) Hģă, H., op. cit., p. 281-285.

(15) id., p. 199.

(16) Zbiss, S.-M., Les monuments de Tunis, STD, 1971, p. 62. As sarraj, El hulal, edité par Hila, M. H., Tunis, STD, 1970, p. 1.089 . 
dans les cimentières communs, soit éparpillées dans toute la ville arabe ou medina.

Dans les cimetières communs, les tourbas sont découvertes; en général c'est un enclos (Huḍa) délimité par un petit mur et reservé aux membres d'une même famille (17). Au cimetière du Gorjani, par exemple, une aire atait reservée aux princes et princesses hafsides (18). Au Zelliji, il existe plusieurs tourbas du même genre (19), signalons au passage celle d'al kurtubi (20).

Les tourbas hors des cimetières, par contre, sont couvertes. Nous pou-

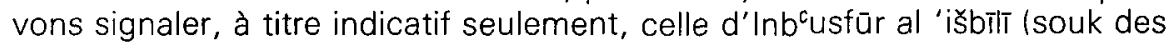
etoffes) (21).

Sur le plan architectural, ces tourbas de la Medina de Tunis, se carecterisent par leur toiture pyramidale à tuiles vertes comme celles d'el Hamra; celle de Youssef Dey (VXII e siécle) est I'oeuvre de l'andalous Ibn Gälib (22); $d^{\prime}$ 'autres tourbas ont une coupole hémispherique bulbeuse à tuiles vertes et dont les murs sont revêtus de stuc ciselé à la manière grenadine (23).

Evidemment, ces tourbas comportent une cour à ciel ouvert surlaquelle s'ouvrent, sous les portiques, des chambres sépulcrales où s'alignent des tombes. La tourbet el bey (rue du même nom) est la necropole des souverains husaynites (1705-1957) (24). Son décor ne diffère pas des autres bien que son architecture soit plus compliquée.

\section{II les cimetières communs (makāāir ou jababin)}

Généralement les cimetières musulmans se trouvent aux abords des villes, ou aux débouchés de ses portes et portent dans ce cas le nom de ces portes (bāb). Donc la plupart de ces champs funeraires sont à la limite de l'agglomeration urbaine; c'est à dire endehors des remparts (25). II en existe aussi à l'interirur de cette agglomeration comme nous allons le voir pour le cas de Tunis.

Un point essentiel reste à savoir la difficulté pour le chercheur de délimiter d'une manière précise non seulement la superficie mais aussi les contours et l'emplacement exact (26).

(17) Al Kinani, op. cit., p. 98. Zbiss, S.-M., Inscriptions du Gorjani, Tunis, INAA, 1962, p. 41.

(18) Zbiss, inscriptions du Gorjani, p. 121

(19) Ben Mami, op. cit., p. 17, note 42 .

(20) Huğă, H., op. cit., p. 186.

(21) Ar rașaac, op. cit., p. 124.

(22) Zbiss, monuments de Tunis, p. 47.

(23) Id., p. 48

(24) Id., p. 50

(25) Brunschvig, R., Berberie orientale sous les hafsides, Paris, Maisonneuve, 1940, I, 354. Zbiss, note..., p. $1,2$.

(26) C'est le cas par exemple du cimetière es silsila qui s'etend entre la Kasbah et Bab Jedid pour Maamouri, 
Pour ce qui est de Kairouan, par exemple, c'est au Nord et à l'Ouest que se répandent les grands cimetières (27) d'Al Balawiyya (28) applée aussi ar rayhāna (29).

Dans le Sud, du coté de l'ancien Bab ar rabí a, c'est le cimetière d'al 'Assäl (30). A l'est de la ville et pret de la porte Nāfica se trouvent les cimetières de Sahnnóun et des Suyūrūn (31).

\section{cimetières de Tunis \\ A Intra muros}

\section{1 cimetière Sidi Bou Khrissan}

Il est situé juste contre Bab menara; il s'etend de la Kasbah jusqu'à la mosquée el Ksar-il est appelé plus tard Sidi Bennfis et puis es silsila; c'est la necropole des Banu khurassan (XI-XII e siècles); c'est aussi le plus ancien que I'on connaisse vu que le cimetière initial de Tunis demeure inconnu 'cette nécropole a continué à recevoir les morts jusqu'an début de ce siècle (32); non loin de ce cimetière se trouve celui de Mhanna à l'est de bāb yantajmi (actuellement souk desetoffes) où et enterré lbn 'usfür al išbilī comme nous l'avons signalé precédemment (33). Dans ce cimetière, il ne reste que la zaouia de Sidi Abdallah at turjuman (Anselm Turmeda) le majorqain (34).

\section{La kasbah}

$C^{\prime}$ est le cimetière de quelques souverains hafsides et ottomans (35) appelée aussi makbarat aš šaraf. Non loin de ce dernier se trouve celui de Sidi Ben Ziad (36).

\section{3 cimetières de Sĩdicay àd, al Manātki et Temīmī}

Ces cimetières se trouvent contre Bab jedid; le dernier comporte la tombe d'un valencien (37).

p. 178, note 65 alors que pour Zbiss: «ll est occupé aujourdh'ui par le parc de l'Hopital Sadiki» (Inscriptions de Tunis, p. 49 , note 55 bis.

(27) Brunschvig. R., op. cit., 1, p. 371.

(28) Roy, B., Poinssot, P., Inscriptions arabes de Kairouan, Paris, Klincksieck, 1950, p. 220, 234.

(29) Al Kinani, op. cit., p. 58, 101-103.

(30) Brunschvig, R., op. cit., 1, 371. Zbiss, Nouvelles inscriptions de Kairouan, Tunis, INAA, 1977, p. 21.

(31) Brunschvig, op. cit., I, p. 371. Roy, B., op. cit., p. 210, 278. Kairoan compte d'autres cimetiéres Sīdĩ Arfa ou rammãdia (Roy, p. 130), At tabbān (ld., p. 146) et al Ğanāḥ al ahudar (Zbiss, nouvelles inscriptions de Kairouan, p. 21.

(32) Zbiss, Inscriptions de Tunis, p. 45. Id. note, p. 2, 4

(33) AR rașșac, op. cit., p. 124, note 21.

(34) Ben Mami, p. 10. Epalza, situación de la tumba de Turmeda.

(35) Ithaf, chapitre VI, edité par A. Abdessalam, Tunis, Université de Tunis, 1971, p. 225. Mass'udī, op. cit., p. 54, 78, 103.

(36) Zluss, note, p. 4

(37) Id., p. 4. Al Hulal, p. 1.027. 
4 cimetières Sidi Younis (rue Rakkah) Sidi Halaf (Rue Sid Mohriz), Sidi Mohriz (contre la zaouia du même nom) et al halfawine. Ces cimetières etaient situés du coté de bab swika (38).

\section{B cimetières extra muros \\ 1 Faubourg Nord}

Bab aluj: cimetières tubkhnāna et sidi Saḳa.

Bab Saadoun: cimetière al Faddān.

Bab Sidi Abdessalam: cimetière du même nom.

Bab al 'Asal: cimetière al bașili (39).

Bab al khadra: cimetière Sidi 'Abd Al Hak et sidi Soufiane. C'est le cimetière des andalous où est enterré le dernier des Abencerrage qui joua un grand rôle dans la cour de Grenade avant sa chute (40).

\section{Faubourg Sud}

\section{Bab al Jazira}

cimetière du Gorjani: c'est le cimetière des princes et des personnages d'etat almohade et hafside. Les epitaphes s'etendent de l'année 1098 à 1494 (41).

\section{cimetière Sidi Mansour (42)}

cimetière du Zellaj: c'est le cimetière de Tunis par excellence. Plus de 300 savants y sont enterrés. Notons, au passage les tombes de deux andalous al Kurțubĩ (43) et aṣ Șarakustī (44) et d'autres (45).

\section{Bab Jedid}

cimetière du Gorjani: appelé aussi al maḳbara al hintātiya ou Saraf al Mourkad (46).

\footnotetext{
(38) Id., p. 5. Ben Mami, op. cit., p. 10.

(39) Ben Mami, B., op. cit., p. 10.

(40) Epalza, M., R., Petit, op. cit. p. 268. Gafsi, A., note sur l'archeologie: source de l'histoire morisco andalouse, in actes du II Symposium International du C.I.E.M., Tunis, 1984, I, 307.

(41) Zbiss, Inscriptions de Tunis, p. 56. Id. Inscriptions du Gorjani, p. 4. Id. note p. 3.

(42) Huğa, H., op. cit., p. 210.

(43) Ben mami, B., op. cit., p. 10

(44) Huğa, H., op cit., p. 187.

(45) Nous sommes en train d'etudier les stéles funeraires decouvertes par Zbiss dans ce cimetiere en compagnie de cedernier et de Boughanmi, $M$.

(46) Zbiss, note, p. 6.
} 


\section{Bab Hāilid}

cimetières de Sidi Kasim ez zeliji et Sidi Bouḥmāma (47).

Il apparaît à l'evidence que nous n'avons fait que schematiser cette partie de l'exposé, car il ne serait pas utile, à mon sens, d'énumerer les differentes tombes et de suivre l'evolution de chque cimetière, d'autant plus que seul le cimetière du zellaj subsiste.

Nantis de ces considerations d'ordre méthodologique, il convient à present d'essayer de dégéger quelques aspects des stèles funeraires.

\section{Tombes et stèles funéraires}

Dans les cimetières communs, les tombes peuvent être soit des tertres de terre avec une pierre anonyme sur la tête, soit des coffres construites en briques ou en pierre, sous forme de deux marches superposées et badigeonnées à la chaux de coulerur blanche ou bleue (48).

Quelques unes par contre sont enrichies de dalles de marbre ou de carreaux de céramique, alors que sur la tête se trouve une stèle gravée d'inscription. D'autres sont couronnées d'une coupolette en miniature.

Dans les tourbas, les tombes (tourbet el Bey) par exemple, sont des coffres en marbre ornées de motifs bas reliefs, au dessus desquelles se dressent des cippes ou des colonnes coiffées de turbans ou de tarbouch pour differencier les deux sexes (49).

Pour ce qui est de la tombe du saint, elle est cachée par un catafalque (tābūt) en bois ouvragé ou en moucharabie et couverte d'ex-votos: etendards, etoffes, tapis, textes sacrés, images de la Mecque...

\section{Disposition et orientation}

Bien entendu, ces tombes, s'alignent côte à côte et sans aucune disposition particulière en général, mais l'orientation est toujours la même.

En effet, d'aprés la règle canonique musulmane, le mort doit être enterré sur le flanc et en face de la Mecque, c'est-à-dire, elle doit être perpendiculaire à la Kibla (50). Cette orientation peut signifier à mon sens l'unité du monde musulman par rapport au monde divin, mais aussi un rappel aux vi-

(47) Id., p. 7.

(48) Cette couleur se repand surtout à Bizerte, Hammamamet et Hergla

(49) Marçais, G., Manuel d'art musulman, Paris, Picard, 1927, II, p. 868. Zbiss, Monuments de Tunis, p. 47.

(50) Vatin, N., Note sur le rôle de la stèle funeraire et l'aménagement des cimetières musulmans à Istanbul, RHM, 47-48, 1978, p. 297. 
vants que la Mecque est à la fois dans les deux mondes des vivants et des morts et que le lien entre les deux est trés étroit.

On doit noter aussi que le terme Kabre (tombe) est presque exclusivement employé en epigraphie funeraire alors que le terme (laḥd) sépulcre est trés peu courant (51).

\section{Forme des stèles:}

Actuellement, seule la plaque rectanguiaire et employée. Mais les ovrages epigraphiques cités plus haut, nous revélent d'autres formes comme la stèle prismatique ou lame (saif) avec base moulurée, colonnette sormontée d'un dôme outrepassé (52) stèle discoïdale (53) ou dalle posée ou scellée horizontalement. Cette dernierè forme apparaît d'aprés S M Zbiss, à partir du XIII e siècle; elle est décorée d'un reseau de marqueterie ceramique qui semble être une innovation andaluose (54).

Bien entendu, les stèles sont en marbre ou en pierre calcaire (Kaddal). Mais peut on parler d'atelięr officiel dans la confection de ces stèles comme le suggère, notre mâ̂tre, Zbiss (55).

\section{Ecriture et décor}

Les stèles comportent un certain nombre de lignes de koufique ou de cursive (56) parfois cette cursive est andalouse (57); ces lignes peuvent etre séparées par des listels (58) cette ecriture peut être ou bien en creux ou en relief, fleurie et parfois non diacritée (59).

Sur le plan décoratif, les lignes sont coiffées parfois d'une arcature (60). Mais ce sont les stèles de forme prismatique qui comportent un décor plus recherché, en effet les bases de ces stèles peuvent etre ornées de tresses, de chevrons, de rinceaux, de palmettes, de perles, de pirouettes, d'épis ou de fleurs $(61)$.

\footnotetext{
(51) Zbiss, Inscriptions du Gorjani, p. 116. La pratique de l'incineration est aussi utilisée mais uniquement comme moyen de vengeance. Ben Mami, op. cit., p. 22.

(52) Zbiss, Nouvelles inscriptions de Kairoauan, p. 58.

(53) Voir Photos et Macsudi, op. cit., p. 64.

(54) Zbiss, inscriptions du Gorjani, p. 11.

(55) Id., Inscriptions de Tunis, p. 70.

(56) Notre article, muhāwala lidirāsat båḍ కawăhid kūbūr an dalusiyy a Mina az zalıāj, $10^{e}$ congres d'archéologie arabe, Tlemcen, 15-18 novembre 1982 (sous presse).

(57) Zbiss, Inscriptions de Tunis, p. 71.

(58) Id., Inscriptions du Gorjan, p. 30.

(59) Id., Gorjani, p. 8.

(60) Id., nouvelles inscriptions de Kairouan, p. 33.

(61) Id., Inscriptions de Tunis, p. 76. Id., Inscriptions de Kairoan, p. 36, 53, 67, 72.
} 
Bien entendu le decor employé dans les stèles merite une etude plus détaillée et plus profonde.

\section{Contenu}

En général, chaque stèle contient des formules pieuses (surtout la formule de profession de foi islamique, la šahāda), des citations coraniques (62), le nom du défunt, sa kunya et sortout une date en toute lettre ou en chiffres; cette date qui termine l'epitaphe (63) lui confère un interêt historique, sociologique, économique et artistique irremplaçable. C'est ainsi que nous avons pu dénombrer un certain nombre de metiers et de professions (64). Quelques stèles portent un texte poétique (65).

\section{Lieu d'exposition:}

Les stèles découvertes en grande partie par notre maître, Zbiss, sont actuellement exposées aux musées de Sidi Bou Khrissan (66), Le Bardo, Zaouia de Sidi Kasim ez zeliji à la Kasbah de Tunis et au ribat de Monastir.

\section{Conclusion:}

En conclusion nous pouvons dire que malgré l'effort fourni par $M$. Zbiss dans ce domaine un inventaire géneral des stèles funeraires s'impose.

En nous traçant la voie à suivre et surtout en dégageant des centaines de stèles et en les conservant dans des conditions extremement difficiles, il merite toute notre reconnaissance.

II nous reste encore à dégager un certain nombre de problèmes: emplacement, évolution, integration des cimetières dans le tissu urbain. L'affectation ethnique demeure encore en suspens. En effect nous savons que quelques cimetières etaient affectés à un groupe ethnique determiné comme c'est le cas de celui des kouraychites à Kairouan et à Cordoue (67).

Pour terminer, citons une meditation tres significative de R. Ricard:

«lls (les cimetières) n'ont pas la froideur et la tristesse des cimetières chretiens. Si quelque mélancolie les enveloppe, ils ne sont jamais dénudés de poesie. Leur situation est telle que on y jouit d'une vue souvent trés belle, propice

(62) Nous avons dénombré pas moins de 30 formules et citations coraniques: Coran, IX, 33. XVI, 53. XVIII, 39. XII, 112. XXI, 34, 35. XXIV, 36, 37. XXX, 4, 5, 6, 7. XXXIII, 21. XXXVIII, 67, 68. XL, 44. XLIV, 51 à 59. CXII, 112. II, 255. 285, 286. III, 185, 173, 174. IV, 78, 135, VII, 54, 55.

(63) Zbiss, Inscriptions de Tunis, p. 52.

(64) Id., Inscriptions de Tunis, p. 76

(65) Id., Nouvelles inscriptions de Kairouan, p. 45.

(66) id., Nouvelles inscriptions de Kairouan, p, 21.

(67) Id., Note, p. 1, 2. Al H.lal, p. 255. 
à la rêverie et à la contemplation. Trés visités par les vivants, qui aiment à mediter auprés des morts, ils sont un lieu de promenade trés frequenté» (68).

(68) Ricard, P., Pour cemprendre l'art musuiman dans l'Afrique du Nord et en Espagne; Paris, Hachette, 1924, p. 216. 


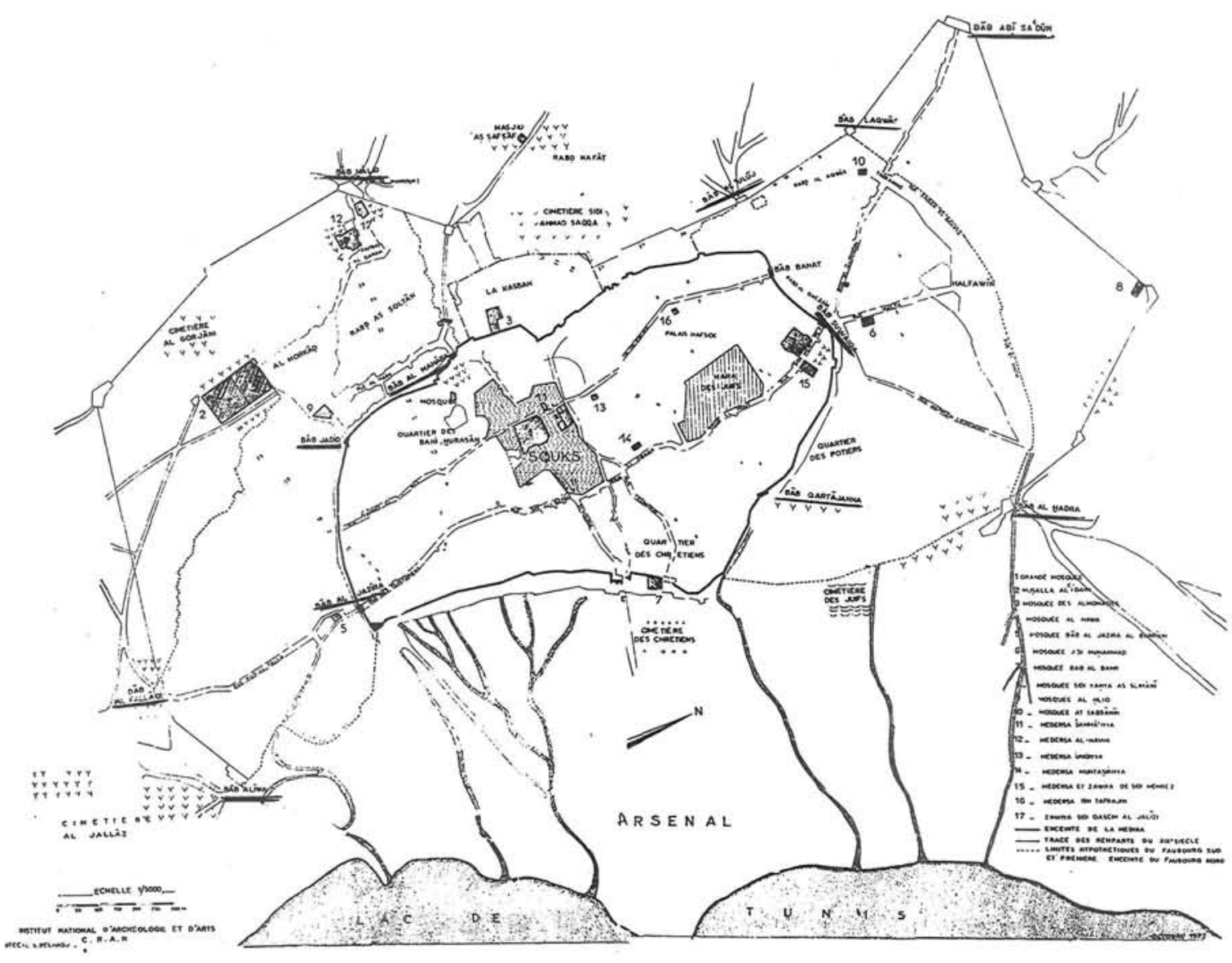

Fig. 1.-Plan général de Tunis aux $X \mid 11^{\ominus}-X V 1^{e}$ siècles, selon A. Daoulatli, avec la situation des cimetières, près des portes de la ville. 


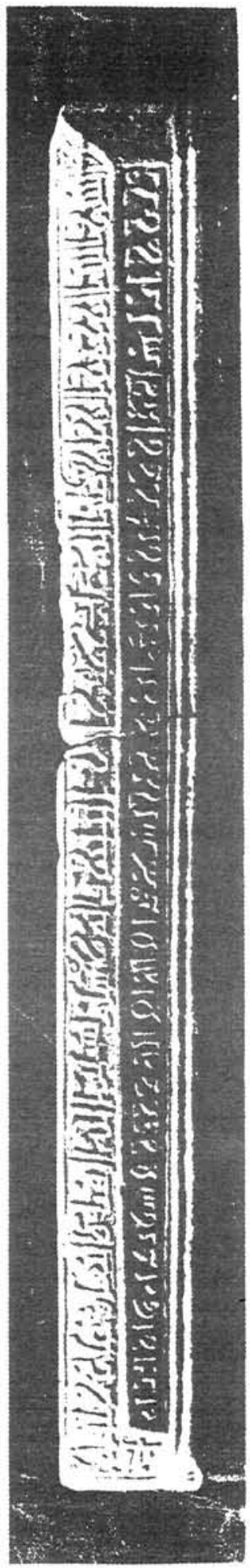

NOTE SUR LES CIMETIÈRES EN TUNISIE / EI Gafsi
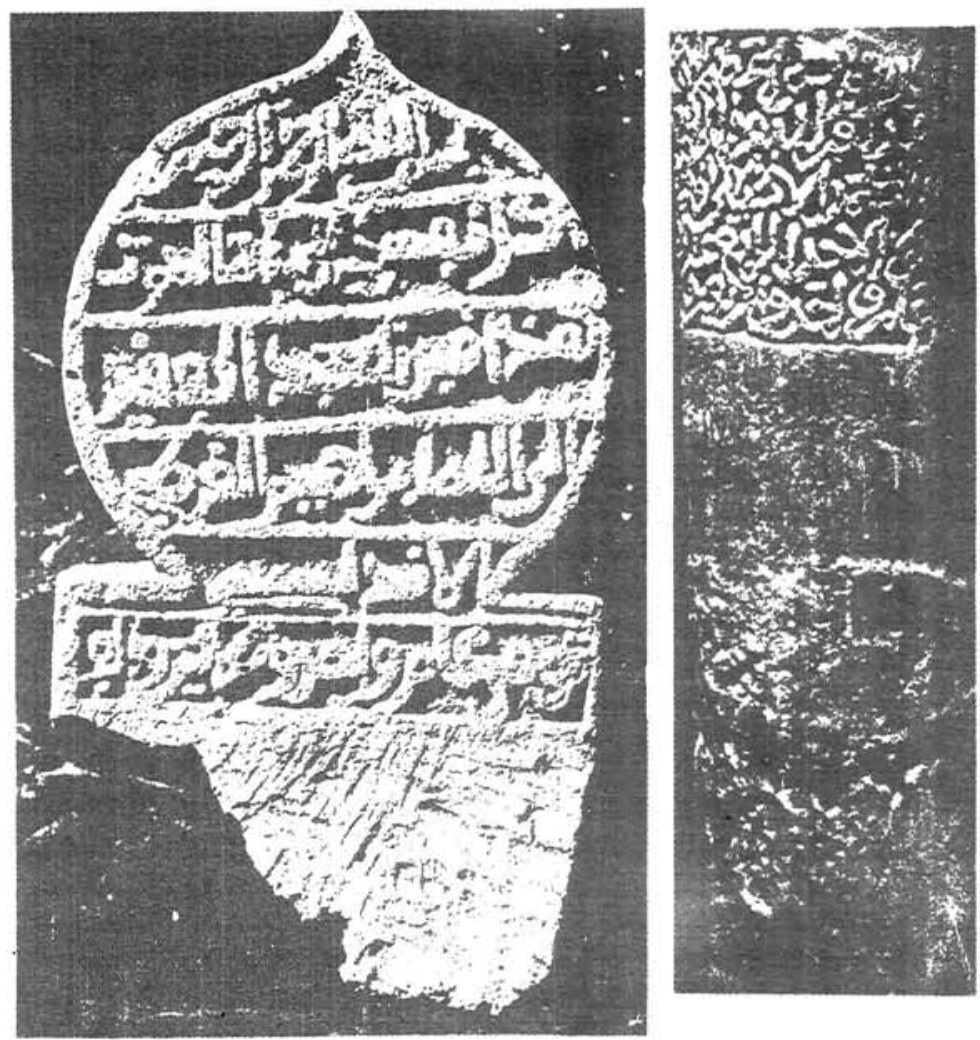

Fig. 2.-Diverses inscriptions funéraires tunisoises. 